\title{
Métaphores, déterminations et temporalités en histoire des techniques
}

De la mécanique du point à la psychologie des nénuphars

Metaphors, determinations and temporalities in history of technology. From the point mechanics to the psychology of water-lilies

\section{Pierre Teissier et Anaël Marrec}

\section{(2) OpenEdition}

\section{Journals}

\section{Édition électronique}

URL : http://journals.openedition.org/artefact/5012

DOI : 10.4000/artefact.5012

ISSN : 2606-9245

\section{Éditeur :}

Association Artefact. Techniques histoire et sciences humaines, Presses universitaires du Midi

\section{Édition imprimée}

Date de publication : 15 décembre 2019

Pagination : 309-338

ISBN : 978-2-8107-0667-9

ISSN : 2273-0753

\section{Référence électronique}

Pierre Teissier et Anaël Marrec, « Métaphores, déterminations et temporalités en histoire des techniques », Artefact [En ligne], 11 | 2019, mis en ligne le 27 novembre 2020, consulté le 29 novembre 2020. URL : http://journals.openedition.org/artefact/5012 ; DOI : https://doi.org/10.4000/ artefact.5012

\section{(c) (i) (9)}

Artefact, Techniques, histoire et sciences humaines est mise à disposition selon les termes de la Licence Creative Commons Attribution - Pas d'Utilisation Commerciale - Pas de Modification 4.0 International. 


\title{
Métaphores, déterminations et temporalités en histoire des techniques
}

\author{
De la mécanique du point à la psychologie \\ des nénuphars
}

Pierre Teissier et Anaël Marrec

\section{Résumé}

Larticle interroge l'usage de quatre métaphores en histoire des techniques : trajectoire, transition, lignée et système. Il analyse leurs ressorts explicatifs et leurs limites sémantiques à partir de leur domaine source : les sciences de la nature et de l'ingénieur. La déconstruction de certaines évidences historiographiques est suivie par la fabrication de deux nouvelles métaphores porteuses d'autres modes d'appréhension du processus historique : l'histoire des énergies comme « sac de nœuds » et l'histoire comme « psychologie des nénuphars ». L'analyse sémantique permet de proposer une épistémologie de l'histoire selon cinq types de détermination (mécanique, thermodynamique, biologique, cybernétique, psychologique) et cinq formes de temporalité (opératoire, statistique, mémorisée, socioculturelle, intime). Ces déterminations et ces temporalités sont entremêlées dans le processus historique mais leur analyse typologique structure l'intelligibilité du récit historique.

\section{Mots-clés}

épistémologie des sciences humaines et sociales, histoire des énergies, historiographie, sciences de la nature et de l'ingénieur, sémantique

95 Pierre Teissier et Anaël Marrec, « Métaphores, déterminations et temporalités en histoire des techniques », Artefact, 11, 2019, p. 309-338. 


\section{Metaphors, determinations and temporalities in history of technology}

From the point mechanics to the psychology of water-lilies

\section{Abstract}

The article examines the use of four metaphors in the history of technology: trajectory, transition, lineage and system. It analyses their explanatory strengths and semantic limits from their source domain: natural and engineering sciences. The deconstruction of several historiographical evidences is followed by the production of two new metaphors that convey other ways of understanding the historical process: history of energies as "a bag of knots" and history as the "psychology of water-lilies". Semantic analysis allows to propose an epistemology of history according to five types of determination (mechanical, thermodynamic, biological, cybernetic, psychological) and five forms of temporality (operative, statistical, memorized, socio-cultural, intimate). These determinations and temporalities are intertwined in the historical process but their typological analysis structures the intelligibility of the historical narrative.

\section{Keywords}

epistemology of humanities and social sciences, history of energy, historiography, natural and engineering sciences, semantics

\section{Remerciements}

Les auteurs remercient les deux rapporteurs de l'article pour leurs commentaires, leurs critiques constructives, et pour leurs judicieux conseils de lecture sur l'analyse linguistique des métaphores. 


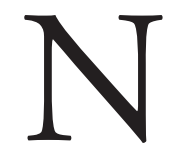
otre article interroge l'usage des métaphores en histoire des techniques, dont une large part provient des sciences de la nature et de l'ingénieur ${ }^{1}$. Le fait est significatif si l'on admet simultanément l'intérêt créatif des transferts sémantiques vers l'histoire des techniques et l'impératif épistémologique d'une autonomie conceptuelle des sciences historiques vis-à-vis des sciences expérimentales. C'est dans cette perspective double, de dialogue et de distinction entre des savoirs différents, que nous analysons l'usage par les historiens des techniques de métaphores scientifiques et techniques. Nous avons sélectionné quatre métaphores largement utilisées en histoire, sociologie ou anthropologie des techniques : trajectoire, transition, lignée et système. Nous analysons les soubassements explicatifs et les limites sémantiques de ces quatre énoncés métaphoriques. Cette déconstruction de certaines évidences historiographiques est suivie par la constitution de deux nouvelles métaphores porteuses d'autres modes de compréhension du processus historique.

La première partie du texte, qui emprunte à la sémantique, discute l'appréhension métaphorique du temps vécu. Elle confronte les complexes spatio-temporels de l'expérience humaine à la conception univoque de l'espace et du temps, selon la mécanique moderne. Les trois parties suivantes détaillent les principales utilités pour les historiens des techniques des quatre métaphores et quelques-unes de leurs limites : dans la deuxième partie, la question de l'inertie en comparant trajectoire et transition; dans la troisième partie, les pratiques mémorielles à travers les lignées techniques ; et, dans la quatrième partie, les interrelations sociales et techniques au sein d'un système. L'analyse critique de ces métaphores suggère un certain nombre de garde-fous contre de fausses évidences : comparaisons floues ; répétitions de mythes collectifs ; sur-évaluation d'homogénéités

\footnotetext{
1. Cette réflexion résulte de notre participation au colloque Face à la puissance. Une histoire des énergies alternatives et renouvelables à l'âge industriel (XIX'-XX' siècles) organisé, en mars 2018, à l'université de Bourgogne par le Réseau universitaire de chercheurs en histoire environnementale (RUCHE) et le Centre Georges-Chevrier. Les organisateurs du colloque proposaient de renouveler l'historiographie à travers plusieurs axes de réflexion dont "une réflexion sur les mots et les catégories utilisées pour penser les systèmes énergétiques, et sur l'évolution du langage pour décrire les choix énergétiques ", notamment les notions de "renouvelable " et d'" alternatif " (Jarrige et Vrigon, 2017). Notre communication, qui analysait quelques machines solaires et éoliennes des années 1940 aux années 1960, concluait à l'absence de corrélation forte entre le type d'énergie utilisée et le projet politique sous-jacent (Marrec et Teissier, 2018). La source d'énergie ne déterminant que très partiellement la dimension politique des techniques, nous en déduisions que la construction de "lignées techniques » selon une même source d'énergie n'allait pas de soi.
} 
temporelles et de cohérences systémiques; etc. Elle permet aussi d'associer chaque métaphore à un type de détermination et à une forme de temporalité différents. Il devient ainsi possible d'articuler, en histoire des techniques, quatre degrés de contrainte : mécanique, statistique, biologique et culturelle. La cinquième et dernière partie revient sur la spécificité des sciences humaines et sociales par rapport aux sciences de la nature et de l'ingénieur. Ce faisant, elle attire l'attention sur l'importance de garder une position distanciée par rapport aux métaphores adoptées car celles-ci transmettent une représentation du monde et du temps. La proposition de deux métaphores inédites en fin d'article clôt l'argumentation par une contribution à l'épistémologie des sciences humaines et sociales.

\section{Métaphores du temps vécu et modèles du temps mécanique}

\section{Le temps vécu d'après la théorie contemporaine des métaphores}

Les métaphores dont nous parlerons sont qualifiées de " conceptuelles " par les linguistes, par opposition aux " expressions métaphoriques linguistiques " qui en découlent et qui les démultiplient à travers la création littéraire notamment ${ }^{2}$. De telles métaphores sont indispensables à l'appréhension de concepts abstraits en termes concrets et imagés. Elles transferrent des mots de "domaines sources » $(\mathrm{A})$ vers des « domaines cibles » (B), qui sont ainsi liés par " un ensemble de correspondances systématiques ». La mise en relation fabrique du lien et de l'écart. Elle " consiste dans un déplacement et dans une extension du sens des mots ${ }^{3}$ ". Mais, comme les métaphores sont constitutives de la manière dont les êtres humains font l'expérience du monde, cette tension devient " conventionnelle " pour la plupart d'entre elles ${ }^{4}$. L'énoncé métaphorique emprunte à un certain nombre de domaines sources qui se prêtent bien aux transferts : le corps humain, la santé et la maladie, les animaux et les plantes, les constructions, les machines, les forces, les mouvements et la direction, etc. ${ }^{5}$. Les trois pre-

2. Kövecses, 2010, p. 4-7.

3. Ricour, 1975, p. 7.

4. Lakoff et Johnson, 1980, p. 126-138.

5. Kövecses, 2010, p. 18-22. 
mières métaphores d'usage courant en histoire des techniques - trajectoire, transition et lignée - proviennent du domaine des « mouvements et direction " tandis que la quatrième - système - provient des domaines « corps humain» et « machines».

Le premier transfert sémantique mérite une attention spéciale car il soustend la représentation " conventionnelle » du temps vécu. Il peut être analysé à travers la " théorie contemporaine de la métaphore » développée par George Lakoff et ses élèves à partir de la fin des années 1970. Leurs travaux identifient un modèle métaphorique central en linguistique contemporaine : la "structure événementielle " (event structure). Ils montrent ainsi que " différents aspects de la structure événementielle, incluant des notions comme états, changements, processus, actions, causes, finalités et moyens, sont cognitivement caractérisés par des métaphores en termes d'espace, de mouvement et de force ${ }^{6}{ }^{\prime}$. Plus spécifiquement, ils insistent sur la manière dont, en anglais (mais également en français), le temps est conceptualisé en termes d'espace, c'est-à-dire en terme " de choses (entités et lieux) et de mouvements ${ }^{7}$ ». Les métaphores de « trajectoire ", «transition " et "lignée " figurent donc les temps comme des objets en mouvement.

Une telle métaphorisation est efficace car « la cartographie n'est pas arbitraire mais s'inscrit dans le corps et l'expérience et la connaissance quotidienne " des individus ${ }^{8}$. Ainsi, ces trois métaphores fonctionnent bien en histoire des techniques parce qu'elles s'inscrivent dans l'expérience vécue du temps par les êtres humains. La représentation du processus historique en termes de "flèche du temps", malgré la conscience de ses limites, relève de cette habitude de vivre et de penser. La deuxième raison de l'usage courant de ces métaphores tient à la position relative des domaines source et cible. La spatialisation du temps sous la forme d'une " trajectoire " renvoie à la construction des sciences modernes à partir du XVII ${ }^{\mathrm{e}}$ siècle et, à la plus mathématisée d'entre elles, la mécanique. Or, comme le souligne Bernard Quemada dans sa réflexion sur "Technique et langage », les " emprunts intertechniques " issus " des contacts et des échanges " entre techniciens sont orientés par des rapports de " prestige » et de "dépendance » entre spécialités et professions ${ }^{9}$. On peut supposer que les emprunts de métaphores

6. Lakoff, 1993, p. 219.

7. Ibid., p. 216.

8. Ibid., p. 245.

9. Quemada, 1978. 
(trajectoire, transition et lignée) aux sciences de la nature s'inscrivent, en partie, dans une stratégie d'accroissement de capital scientifique par les sciences de l'homme.

\section{Un hiatus entre l'espace-temps classique et l'expérience de l'histoire}

Ces emprunts ont une conséquence épistémique majeure pour les sciences de l'homme et de la société. Ils tendent à rendre "naturel " et " universel » le modèle du temps mécanique conçu par les sciences modernes au $\mathrm{XVII}^{\mathrm{e}}$ siècle. Ce temps est linéaire (unidimensionnel), homogène et orienté. Il est couplé à un espace tridimensionnel, vide, illimité et immuable. La puissance scientifique de cet espace-temps est de rendre possible les opérations de calcul et de mesure. En effet, la mécanique classique calcule la " trajectoire " des corps en mouvement ayant une masse, ou " mobiles", à partir de la mesure de leurs coordonnées initiales (position et vitesse) et de la relation fondamentale de la dynamique (accélération multipliée par la masse égale à la somme des forces). Ce faisant, elle considère des relations de cause à effet très fortes. On parle de déterminisme strict parce qu'un corps en mouvement suit une trajectoire prévisible sans écart possible, c'est-à-dire sans degré de liberté. Il constitue le type de "détermination ${ }^{10}$ " le plus contraignant. À la suite de la mécanique, les sciences de laboratoire ont adopté ce temps opératoire. Celui-ci influence également les sciences de la société, qui, après Émile Durkheim, ont été tentées par un physicalisme social. Durkheim insiste d'ailleurs sur l'impossibilité de saisir le temps sans le diviser, c'est-à-dire sans le rendre opératoire ${ }^{11}$. Le temps mécanique permet également d'apprivoiser les "discontinuités " des sciences historiques en les présentant comme des intervalles de temps, identifiables et mesurables, dans un processus continu ${ }^{12}$.

10. Nous prenons « détermination " dans le sens d'une relation entre des facteurs et des effets dans le processus historique. Les formes de détermination sont multiples par le degré de contrainte (pression) et de surgissement (création) qu'elles permettent. Les types de détermination se distinguent par l'équilibre relatif de contraintes et de surgissements, la forme et l'intensité de la relation entre facteurs et effets et la forme des temporalités associées.

11. Durkheim, 1912.

12. «Pour l'histoire dans sa forme classique, le discontinu était à la fois le donné et l'impensable : ce qui s'offrait sous l'espèce des événements dispersés - décisions, accidents, initiatives, découvertes ; et ce qui devait être, par l'analyse, contourné, réduit, effacé pour qu’apparaisse la continuité des 
Une partie de l'historiographie souligne, au contraire, les limites d'une telle représentation. L'ordre du discours foucaldien réfute l'apparente identité de l'auteur, de l'œuvre et de l'objet. Avant Michel Foucault, Walter Benjamin met en garde contre la conception d'un temps homogène et vide, qui soutient l'image d'une histoire universelle de l'humanité en marche dans le sens du progrès ${ }^{13}$. Aux antipodes du temps mécanique, le temps de l'histoire est " hétérogène » et " déformé ", "surpeuplé » de "fantômes » insaisissables dans leur totalité et leur objectivité ${ }^{14}$. Les tenants du temps universel pourraient soutenir, à la suite du modèle de la «structure événementielle ", que la spatialisation du temps dans l'expérience individuelle est conforme au modèle du temps mécanique. Une telle position est plus spontanée que réfléchie. En effet, les travaux de Reinhardt Koselleck montrent que les modes d'appréhension du temps par l'expérience, ou " champs d'expérience ", sont multiples. Il identifie, à partir du vécu individuel et collectif, " trois types de gain d'expérience ${ }^{15}$ ». Il y a d'abord l' "expérience originelle ", associée à " la surprise ", qui, par sa singularité radicale, ne peut être répétée. Ensuite, le gain d'expérience peut opérer par « accumulation " d'expériences, qui, elles, sont non seulement " répétables " mais également " recueillies " par un individu ou une génération. Enfin, le troisième type concerne " une mutation systémique ", qui transforme radicalement " tout le capital d'expérience antérieur acquis à court ou moyen terme ». L'analyse de Koselleck, en densifiant « l'expérience de l'histoire ", diversifie les formes de temporalité historique.

Le temps mécanique apparaît, pour les sciences historiques, comme une "prénotion " au sens de Durkheim, c'est-à-dire comme une idée spontanée et commune. Il s'agit de mettre à distance le temps mécanique pour des raisons anthropologiques puisque l'expérience quotidienne le fait accepter de manière automatique et inconsciente. La raison de la distanciation est aussi historiographique puisque le temps mécanique n'est qu'une des formes de temporalité historique parmi d'autres : temps vécu, champs d'expérience,

événements. La discontinuité, c'était ce stigmate de l'éparpillement temporel que l'historien avait à charge de supprimer de l'histoire. " (Foucault, 1969, p. 16)

13. Benjamin, 2014.

14. « La structure subjective de la durée acquise à l'ère industrielle est celle d'un temps accéléré, d'un temps surpeuplé comme l'exprime joliment René Nelli. Elle ne permet de repérer dans le passé que des fantômes situés dans des espaces-temps déformés, et non des objets que la science en progressant pourrait un jour décrire complètement. " (Gras, 1979, p. 190)

15. Koselleck, 1997, p. 271-280. 
discontinuités temporelles, peuplement des passés et des futurs, etc. Dans les trois prochaines parties, nous analysons la pertinence des systèmes de correspondance des métaphores sélectionnées entre le domaine source, des sciences de la nature et de l'ingénieur, et le domaine cible, de l'histoire des techniques. Nous considérerons successivement la paire trajectoire-transition, puis la lignée technique et enfin le système sociotechnique. L'enjeu est sémantique certes mais, plus fondamentalement encore, épistémologique. Paul Ricœur l'exprime de manière frappante lorsqu'il écrit : " La métaphore, en dernier ressort, est pouvoir de redécrire la réalité ${ }^{16}$ ». Elle transporte donc une représentation du monde et du temps.

\section{Re-présenter l'inertie et la fluidité des sociétés humaines : trajectoire et transition}

L'usage des métaphores de " trajectoire » et de «transition » est courant en histoire des techniques pour rendre présent, ou re-présenter, des fluidités 316 et des inerties du processus historique.

\section{Trajectoire : représentations mécaniques et inerties historiques}

Commençons par interroger la métaphore de la " trajectoire " par rapport au domaine source. En mécanique classique, le mobile a une "inertie». Il s'agit, comme l'écrit Isaac Newton, d'un " pouvoir de résistance par lequel chaque corps persévère, autant qu'il est en lui de le faire, dans son état actuel de repos ou de mouvement uniforme en ligne droite ${ }^{17}$ ". Un mobile garde donc sa trajectoire sauf si de nouvelles "forces imprimées le contraignent à changer ". C'est ce "pouvoir» inertiel qui intéresse les historiens lorsqu'ils importent le concept. Thomas Hughes introduit le concept d' "impulsion technologique " (technological momentum), dans ses premiers travaux, pour rendre compte de la persistance organisationnelle de l'entreprise I.G. Farben dans un contexte politique radicalement transformé : passage de la république de Weimar au Reich nazi. Il souligne une

16. Ricœur, 1975, quatrième de couverture.

17. Les citations de Newton sont extraites de Blay, 1995, p. 43-44. 
correspondance supplémentaire entre histoire et mécanique : "momentum [...] est plus adapté que conservatisme [...] parce qu'il y a une inertie du mouvement aussi bien qu'une inertie du repos ${ }^{18} »$. Il poursuit en qualifiant la détermination technologique de "déterminisme mou " (soft determinism) au même titre que les facteurs économiques ${ }^{19}$. Il considère néanmoins que la détermination technologique augmente à mesure que les " agrégats d'intérêts professionnels " croissent en taille et en nombre (leur momentum augmente d'autant). Cette inertie de mouvement explique donc la « direction "stable des grands systèmes sociotechniques. Il est intéressant de relever deux failles du transfert sémantique de Hughes. Premièrement, il qualifie de " molle " la détermination technologique mais emprunte sa métaphore au plus déterministe des domaines scientifiques : la mécanique. Deuxièmement, il ne poursuit pas jusqu'au bout l'analyse du système de correspondances du transfert car il recourt souvent au momentum sans jamais mentionner la «trajectoire».

Celle-ci est, au contraire, bien définie par Alain Gras : l'image est motivée par la manière dont une technologie "se développe en suivant un chemin spécifique (technological path) sur lequel elle est bloquée ${ }^{20} »$. La trajectoire est induite par une " contrainte [qui] s'impose dans le devenir " et "verrouille sur un certain axe le devenir d'un artefact ou d'un système technique ». Les facteurs contraignants ne sont pas ici techniques mais sociaux. La métaphore est utile pour penser des " bifurcations " du processus historique. Elle justifie ainsi l'écriture d'histoires " autres " par rapport aux récits dominants : des alternatives, des échecs, des perdants, des oubliés, etc. Dans le cas particulier des énergies, la métaphore de «trajectoire énergétique » attire l'attention sur les conditions initiales : disponibilité ou non de sources d'énergie, impulsions politiques en faveur d'une technologie, etc. Il est alors tentant de dessiner la trajectoire des " énergies renouvelables " sous prétexte que ces nains énergétiques vivent au-dessous des bilans énergétiques ou sont placés à côté des types dominants d'énergie de l'ère industrielle.

18. Hughes, 1986, p. 556.

19. Ibid., p. 465.

20. Gras, 2003, p. 16-20. La métaphore de la trajectoire renvoie également au concept de « dépendance au sentier " (David, 1986). 


\section{Transition : changements lents et déterminations statistiques}

Au début du $\mathrm{xxI}^{\mathrm{e}}$ siècle, les discours politiques sur l'environnement sont saturés de l'expression "transition énergétique ». Celle-ci devrait faire passer graduellement les sociétés industrielles d'une consommation de masse en hydrocarbures à une consommation d'énergie majoritairement « renouvelable» dans un futur proche. Contre « la révolution [...] rupture brusque, voire brutale, la transition est une inflexion lente, voire douce ${ }^{21}$ ». Certains l'imaginent se dérouler, de manière fluide, sur quelques générations à peine. Ce questionnement actuel influence la relecture du passé à la recherche de transitions énergétiques au Moyen Âge par exemple ${ }^{22}$.

Interrogeons cette deuxième métaphore à partir du domaine source : une " transition " désigne en thermodynamique et en chimie un processus orienté, qui mène d'un état initial à un état final. Elle est soumise à une détermination statistique : une fois l'équilibre atteint, un certain pourcentage d'une population d'atomes ou de molécules est passé dans l'état final tandis que le reste est resté dans l'état initial. Ce rapport de population est fixé par les conditions expérimentales. Il est calculable et mesurable. Une telle considération a deux conséquences, l'une épistémique, l'autre historiographique. Au niveau épistémique, l'usage de « transition » présuppose une détermination statistique du phénomène en jeu. Les acteurs sont alors nombreux, semblables, en mouvement les uns par rapport aux autres, et dans un milieu fermé. Leur comportement est collectivement régi par une corrélation statistique. Le modèle sous-jacent pour penser une telle évolution séculaire est " la transition démographique " qui s’est déployée depuis le XVIII ${ }^{\mathrm{e}}$ siècle sous l'effet de facteurs culturels notamment ${ }^{23}$. Si on ramène la métaphore au domaine cible, rien ne dit que la détermination statistique, qui a structuré la démographie depuis l'époque moderne, est applicable à l'histoire des énergies sur quelques générations durant le siècle à venir.

21. Bouvier et Laborie, 2016, p. 15.

22. Voir, par exemple, la thèse de doctorat en préparation à l'EHESS de Sarah Claire intitulée Mix énergétique, crises et transitions en Europe centrale à la fin du Moyen Âge. La rivière Vltava et son bassin en Bohême XIV-XVt siècles.

23. Courbage et Todd, 2007, p. 31-40. 
$\mathrm{Au}$ niveau historiographique, la métaphore suggère que le processus est orienté, de manière simple et univoque. Or, un processus historique est ouvert, en débat, voire en lutte, car les choix à venir ne sont pas arrêtés mais dépendent de l'arbitrage entre des facteurs techniques, économiques, politiques, etc. ${ }^{24}$. L'usage de la métaphore a alors tendance à " naturaliser » et à " fluidifier » une évolution, qui a priori est indéterminée et pourrait être contradictoire. Un taux de $100 \%$ d'énergie renouvelable signifierait-il la fin du processus historique ? Poser la question montre l'absurdité de suivre la métaphore jusqu'au bout. Dans le même registre historiographique, Yves Bouvier comme Jean-Baptiste Fressoz ont montré que l'expression " transition » valorise la dimension «substitutive " des énergies et masque leur dimension " additive $e^{25}$ ». Or, l'addition d'énergies structure l'histoire des sociétés industrielles à mesure que l'équation "civilisation égale énergie " se construit en idéologie ${ }^{26}$.

\section{Limites des métaphores mécaniques et thermodynamiques}

Les métaphores de " trajectoire " et de " transition » ont quelque valeur heuristique en histoire des techniques. Nous pensons néanmoins que, dans le domaine de l'histoire des énergies, elles apportent plus de confusion que de clarification pour deux ensembles de raisons.

En terme méthodologique, bien souvent, les métaphores mécaniques et thermodynamiques sont utilisées sans analyser les systèmes de correspondance sémantique entre les domaines source et cible. L'un des problèmes des métaphores mécaniques est leur proximité avec l'expérience vécue du temps : un observateur fixe, des temps-objets en mouvement. Or, si un type d'énergie par exemple dessinait une trajectoire au cours du temps, quels seraient le référentiel d'étude, la forme et la vitesse de la trajectoire, l'identification du mobile considéré ? Hughes peut s'appuyer sur un système de correspondances dans le cas des réseaux électriques parce que les mailles du réseau relient l'ensemble des acteurs intéressés. Cela ne fonctionne plus pour des concepts aussi diffus qu'un type d'énergie (solaire,

24. Bouvier et Laborie, 2016, p. 17.

25. Respectivement Bouvier, 2012, p. 23-36 et Fressoz, 2013, p. 173-187.

26. Basalla, 1982. 
nucléaire, charbon, etc.) ou qu'un ensemble d'énergies (carbonées, renouvelables, alternatives, etc.) ${ }^{27}$.

Ceci conduit à envisager, plutôt que les types d'énergie, une focalisation sur les convertisseurs énergétiques. Un convertisseur est une machine qui " transforme économiquement l'énergie brute en des formes utilisables ${ }^{28}$ ". Par sa situation de carrefour, le convertisseur cristallise les aspects matériels mais aussi politiques des systèmes techniques plutôt que la seule source d'énergie et ses propriétés physiques. Il constitue ainsi un " nœud technopolitique ${ }^{29} »$. Serait-il possible de tracer la trajectoire d'un convertisseur à l'assimilant à un mobile en mouvement ? Nous doutons que cela soit satisfaisant car une même appellation de convertisseur peut cacher des différences fortes : une pompe solaire par exemple, malgré la pérennité du nom, est fortement modifiée entre les premiers prototypes du début du $\mathrm{Xx}^{\mathrm{e}}$ siècle en Égypte et ceux déployés au Sénégal durant les années $1970^{30}$. Pour asseoir la métaphore, il manquerait également l'identification d'un référentiel spatio-temporel et le tracé d'une trajectoire historique.

La deuxième raison de notre critique à l'encontre des métaphores méca320 niques et thermodynamiques tient à leurs limites épistémologiques. La trajectoire présuppose la continuité des objets étudiés que ce soit un convertisseur ou un type d'énergie. Qu'est-ce qui serait alors conservé ? L'imaginaire de la mécanique classique présuppose des éléments dont l'existence est contestable dans le processus historique : un temps orienté et linéaire, des objets stables dans le temps, dont il est possible de connaître les qualités et de calculer les quantités avec une précision extrême. Le passage de la mécanique à la thermodynamique ne résout pas non plus le problème. Certes, les concepts thermodynamiques, comme la " transition ", articulent deux types de détermination : mécanique pour régir le mouvement local d'un atome ou d'une molécule ; statistique pour régir le comportement collectif de ces populations. Mais, les lois des grands nombres ont tendance à faire disparaître, dans des moyennes, les phénomènes singuliers. Comment alors observer le surgissement de mouvements sociaux, de

27. Marrec, 2018, p. 687-698.

28. Debeir, Deléage, Hémery, 2013, p. 23.

29. La technopolitique est prise dans le sens des " pratiques stratégiques qui consistent à concevoir ou à utiliser la technique afin de mettre en place des objectifs politiques, de leur donner forme et de les réaliser" (Hecht, 2014, p. 24).

30. Diop, 2016. 
créations culturelles, d'innovations techniques dans ces ensembles réduits à des lois statistiques de comportement ?

\section{Graver des mémoires collectives : tracer et retracer des lignées techniques}

\section{Légitimer le présent par la construction mémorielle de généalogies techniques}

Les acteurs construisent eux-mêmes des « lignées techniques », qui relient, au cours du temps, des machines ou des techniciens. Ces lignées sont construites de manière à aboutir aux activités actuelles. Leur rôle a pour fonction principale de légitimer le présent. Ces récits de mémoire collective distinguent, dans les époques passées, des " héros ", des " grands hommes " qui sont qualifiés de "précurseurs " ou de "visionnaires " parce qu'ils ont $\mathrm{eu}$ " raison trop tôt ".

Cette pratique est répandue dans le champ de l'énergie solaire. Au $\mathrm{XIX}^{\mathrm{e}}$ siècle, savants et ingénieurs font du soleil un candidat industriel alors qu’il était déjà objet d'investigation scientifique. Le passé est convoqué pour trouver des expériences similaires antérieures, des formes de patrimoine ${ }^{31}$. On peut alors trouver des "précurseurs " aussi lointains que l'antique Archimède, qui aurait, au $\mathrm{III}^{\mathrm{e}}$ siècle avant notre ère, incendié la flotte ennemie près de Syracuse grâce aux rayons solaires. Archimède devient ainsi la première figure incontournable de tout "bref historique " qui se respecte. Plus modernes sont les figures solaires de Newton, Lavoisier, Buffon ou Ferdinand de Saussure. Progressivement, à partir de la deuxième moitié du XIX ${ }^{\mathrm{e}}$ siècle, les acteurs eux-mêmes de l'énergie solaire s'ajoutent à cet aréopage : Augustin Mouchot, Charles Tellier, Frank Shuman, Charles Abbot ou encore Félix Trombe.

Àl'inverse, ce sont moins les "grands hommes " que les " grandes machines " qui peuplent l'imaginaire collectif de l'éolien. Â la fin du XIx ${ }^{\mathrm{e}}$ siècle, certains chantres du progrès inscrivent les éoliennes de pompage "américaines " dans une lignée technique issue des moulins à vent "hollandais ", dont l'usage pour la mouture et le broyage du grain est alors assimilé au

31. Pehlivanian, 2014. 
passé. À partir des années 1920, "l'aérogénérateur » incarne une nouvelle modernité qui supplante, et efface dans les discours, l'éolienne de pompage $^{32}$. Ceci construit une filiation directe, à deux siècles de distance, de l'aérogénérateur états-unien au moulin hollandais. Pendant la Deuxième Guerre mondiale, alors que plusieurs États financent la recherche et le développement d'aérogénérateurs, l'ingénieur Charles Monney peut déclamer qu'après " avoir végété pendant de longs siècles sous la forme vétuste qu’a illustrée Cervantès, les moulins à vent semblent se disposer à prendre part à la production d'énergie [électrique] en grande quantité, à l'instar de la vapeur et de la houille blanche ». Aujourd'hui encore, le site internet d'EDF présente un "bref historique de l'histoire du vent " qui associe des moments « fondateurs » séparés de longues absences : cet historique débute en l'an mil, s'incarne brièvement à la fin du XIX ${ }^{e}$ siècle à travers les moulins à vent américains, puis, après plusieurs décennies de disparition, ressurgit en 1957 avec l'éolienne de Nogent-le-Roi. Le point d'orgue de ce millénaire d'histoire humaine est la politique actuelle d'installation d'éoliennes offshore par $\mathrm{EDF}^{33}$.

\section{Limites et leçons des lignées techniques : mémoire et " évolution créatrice "}

Que les acteurs légitiment leurs actes par des récits mémoriels grandiloquents n'a rien de surprenant. Ce qui l'est plus est que certains historiens sont tentés de reprendre, sans beaucoup d'investigations, ces généalogies techniques. Comme pour les acteurs, ils peuvent y trouver une forme de légitimité. Mais, ces lignées techniques contribuent, si elles sont manipulées trop rapidement, à favoriser une lecture " évolutionniste ${ }^{34}$ ». Pour éviter cet écueil, il est intéressant de remplacer les lignées techniques par une interprétation des dynamiques techniques comme " modes d'assemblage » de techniques et d'objets ${ }^{35}$. Le concept d' « innovation " est alors décalé au profit d'une " recomposition " permanente des choses et des

32. Marrec, 2018, p. 196.

33. https://www.edf.fr/en/node/194645.

34. Le tracé de "lignées techniques » a d'abord été une pratique courante en anthropologie évolutive pour rendre compte, comme pour les lignées d'espèces vivantes dans la théorie de l'évolution biologique, de lignées culturelles dans l'histoire de l'humanité. C'est un outil largement utilisé par André Leroi-Gourhan, qui est ensuite conceptualisé par Gilbert Simondon.

35. Grimaud, Tastevin, Vidal, 2017, p. 14-15. 
humains : le wild tech peut être pensé en sus des traditionnels low et hightech et les usagers sont aussi légitimes à agir que les producteurs.

Les lignées, comme les trajectoires, engagent à rechercher, dans le passé, des " bifurcations ", à repenser les "échecs " de l'innovation ${ }^{36}$ et à ouvrir des « lignées techniques négligées et des potentialités non advenues ${ }^{37}$ ». Ces approches, outre des enjeux écologiques ou économiques, portent l'empreinte du "paradigme énergétique » caractérisant les sociétés industrielles contemporaines à l'aune duquel sont appréhendées des sociétés anciennes ou autres. Alain Beltran parle même d'homo energicus pour définir l'acteur ordinaire de l'histoire alors même qu'il souhaite souligner qu'il n'est pas "réductible à quelques équations ${ }^{38}$ ".

Cette " histoire à rebours " est sans doute un moyen d'appréhender une histoire complexe. Dans le cas du soleil, son omniprésence et omnipotence sur terre suggère des histoires multiples du " champ solaire ${ }^{39}$ ». La grande variété d'objets et de formes solaires rend son histoire extrêmement vaste et difficile à circonscrire. L'énergie solaire, condition de l'évolution biologique, est utilisée depuis des temps immémoriaux en agriculture, dans les domaines architecturaux ${ }^{40}$ ou pour la mesure du temps. Qu'est-ce qui justifie l'association d'objets aussi étrangers que miroirs ardents et panneaux photovoltaïques $^{41}$ ? Des entrées plus restreintes sont aussi possibles selon les modes de conversion (actif ou passif), les types d'usage (chauffage ou force mécanique), les lieux d'implantation (satellite ou architecture), les technopolitiques (politiques publiques ou R\&D militaire), etc. ${ }^{42}$. Si les historiens du solaire ont tenté de s'affranchir des récits des acteurs, les histoires de l'éolien ressemblent souvent à celles des acteurs du domaine : elles retracent à l'envi des filiations entre aéromoteurs en soulignant l'évolution technique de l'un à l'autre ${ }^{43}$. Ces filiations alternent continuités et ruptures. Elles favorisent l'usage de métaphores comme " histoire à éclipse ${ }^{44}$ ». Si elles présentent un caractère heuristique, les lignées solaires et éoliennes

36. Lamard et Stoskopf, 2018, p. 9-19.

37. Fressoz, 2013, p. 178.

38. Beltran, 2018, p. 308.

39. Boistel et Le Gars, 2015.

40. Chauvin-Michel, 2012.

41. Kryza, 2003.

42. Notamment Herléa, 1995 et Bouvier et Pehlivianian, 2013.

43. Voir par exemple Hills, 1996 et Righter, 2008.

44. Pehlivanian, 2014. 
ont des limites historiographiques : une ambition exhaustive et holiste ; un rapprochement exagéré au nom des "types d'énergie »; une ignorance des spécificités propres à une époque.

Quelle leçon peut-on tirer de l'analyse de la métaphore ? Comme pour les deux premières métaphores, la " lignée " fait pénétrer dans un nouvel espace scientifique - le vivant - et suggère un nouveau type de détermination. Dans la théorie darwinienne de l'évolution, la détermination se situe au niveau d'une dynamique par essais et erreurs, qui n'est pas exempte de créativité et de surgissement. Nous suivons ici Henri Bergson, qui, dans L'Évolution créatrice, situe le surgissement créatif dans l'expérience de la durée par les êtres vivants ${ }^{45}$. Qu'elle soit individuelle ou collective, la mémoire animale constitue une source de créativité. De la même façon que le passage de la mécanique à la thermodynamique avait enrichi le temps mécanique d'une temporalité statistique, l'entrée dans les sciences du vivant définit une forme de temporalité plus complexe, biologique.

\section{Connecter des objets, des hommes et des réseaux : la métaphore du système Caractéristiques du concept de "système "}

Le concept de « système » est utilisé de manière croissante à partir des années $1940^{46}$. La cybernétique, notamment, le convoque pour rendre compte d'interactions hommes-machines dans le cas d'ensembles techniques de contrôle, de régulation et de communication d'informations. Le système est vu comme un moyen de dépasser les déterminations mécaniques (cause à effet) et statistiques (corrélations). La métaphore est issue du domaine du vivant, auquel elle emprunte deux caractéristiques : son approche holiste des interactions entre organismes et milieux; ses références aux fonctions à réaliser, notamment des organes dans un organisme. Les historiens des techniques suivent les ingénieurs dans cette approche systémique. Dans Le Système technicien, Jacques Ellul identifie cinq caractéristiques d'un "système technique ": un réseau d'interrelations de nature et d'intensité différentes; une préférence des éléments du système à se combiner en interne ;

45. Herring, 2016.

46. Le Moigne, 2003. 
une aptitude pour le mouvement (dynamique et innovation); des boucles de feed-back ou "structures de renvoi " au sein du système ; une mise en relation avec d'autres systèmes ${ }^{47}$.

La métaphore du "système " est utile pour l'analyse des techniques. Elle permet d'assembler les cinq critères identifiés par Ellul, de souligner la dimension complexe du sociotechnique et d'articuler les effets contraignants (structures) et innovants (créativité). Elle porte également les images d'organisation a priori et d'auto-organisation. Elle souligne, enfin, les dynamiques de reproduction interne ou " autopoïese " (Francisco Varela, Niklas Luhmann) et "la constitution d'un espace de référence interne ${ }^{48}$ ». Cependant, le modèle du "système " tend à surestimer plusieurs qualités des ensembles techniques : puissance, intégration et logique. Le "système technicien " de Jacques Ellul comme le « techno-cosme » de Gilbert Hottois présentent des ensembles techniques totalisants et totalitaires. Ils négligent ou réfutent la possibilité d'un en-dehors du système, auquel il relie, plus ou moins lâchement, toutes les entités socioculturelles. L'humain, dans ses indéterminations, ses hésitations, ses conflits, semble en avoir complètement disparu. Les autres formes de médiation avec le monde (théorique, artistique, religieuse, politique, etc.) sont écrasées par la force opératoire des techniques. Parler de "système technique " tend à réifier l'ensemble comme un organisme cohérent et autonome, justifié par ses effets fonctionnels efficaces. Le surgissement créatif, les relations passionnées aux mondes et aux êtres, les façons de vivre ensemble sont alignés sur le pas technicien. Sont lissées les hétérogénéités sociotechniques : numérique-analogique, artificiel-vivant, culture-nature, harmonie-conflit, nécessité-liberté, etc.

\section{Dynamique des systèmes : innovations et contrainte sociales}

Bertrand Gille, au contraire, insiste sur « l'interdépendance » entre la technique et les autres activités humaines en société : économique, linguistique, sociologique, politique, juridique, scientifique ou philosophique ${ }^{49}$. Ces interactions sont à la fois " coordination des activités humaines »

47. Ellul, 2012, p. 88.

48. Gras, 1997, p. 84.

49. Gille, 1978, p. viII. 
et disjonctions entre des domaines aux logiques spécifiques. Le système permet alors de proposer " une histoire en quelque sorte enchaînée par le monde matériel [... où] la liberté de l'inventeur est étroitement circonscrite, étroitement limitée par les exigences auxquelles doit répondre l'invention [... par les] éléments les plus contraignants du progrès technique $^{50}$ ». La dynamique des systèmes se comprend comme une tension entre " déséquilibres internes à la technique, cohérences nécessaires entre les diverses techniques, compatibilités avec les autres systèmes ${ }^{51}$ ».

Poursuivant cette réflexion, Hughes parle de «tissu sans couture de composants en interaction " pour souligner l'imbrication contraignante des acteurs institutionnels et des réseaux techniques ${ }^{52}$. Il conçoit la dynamique des systèmes comme l'identification et la résolution de "problèmes critiques ». Il l'analyse, non pas par la métaphore mécanique du "goulot d'étranglement ", mais par l'image du "saillant-rentrant " emprunté au domaine militaire. Cette nouvelle métaphore déploie l'innovation technique sur un territoire par analogie avec un champ de bataille : un front progresse (ou régresse) à travers la formation de " poches avancées " (ou reculées) qui sont des points saillants (ou rentrants). Ainsi, les «bâtisseurs de système " identifient un " rentrant " comme " le point le plus faible " de l'ensemble technique qu'il s'agit de "renforcer ${ }^{53}$ ». Si Gille s'attache à comprendre la succession des systèmes sociotechniques, de l'artisanal vers l'industriel, Hughes forge un modèle de construction des systèmes contemporains en six étapes : création, transfert, croissance, compétition, contingence, inscription ${ }^{54}$.

La métaphore du "système " introduit un niveau supplémentaire de complexité dans l'argumentation. Elle articule les effets déterminants et innovants d' "artefacts culturels en évolution ${ }^{55}$ ". Ces ensembles d'éléments hétérogènes sont organisés et finis, ouverts et en interaction avec d'autres ensembles, eux-mêmes hétérogènes, organisés, finis et ouverts. Leur spécificité la plus remarquable est une aptitude à l'auto-reproduction et à l'émergence de nouveauté par les interactions hommes-machines. La

50. Ibid., p. 45-48.

51. Ibid., p. x.

52. Hughes, 1998, p. 857.

53. Ibid., p. 842-846.

54. Respectivement Gille, 1978, p. Ix et Hughes, 1986, p. 15-16.

55. Hughes, 1986, p. 465. 
dynamique des systèmes sociotechniques est soumise à un nouveau type de détermination, qui redouble "l'évolution créatrice " d'une culturalité créatrice. Ce quatrième type de détermination touche «à la spécificité de l'homme au sein de cette nature [...] en évolution » : « le fait de [produire] de la société pour vivre $[\ldots]$ donc de la culture $[\ldots]$ de l'histoire ${ }^{56}$. Du temps mécanique aux temps statistique et évolutionniste, nous passons au temps socioculturel.

\section{Imaginaires et intelligibilités en histoire des techniques}

L'analyse des usages en histoire des techniques de quatre métaphores scientifiques et techniques nous conduit à formuler deux conclusions partielles et à proposer deux métaphores inédites. La première conclusion partielle concerne plus directement l'histoire des énergies. La discussion des métaphores de trajectoire et de transition a relativisé la cohérence des types de source d'énergie. L'analyse des lignées techniques a souligné la faiblesse historiographique du concept de " trajectoire énergétique " et la dimension mythologique de certaines généalogies en histoire de l'énergie. La discussion sur les "systèmes techniques ", tout en montrant l'importance des interrelations entre éléments hétérogènes, a mis en doute les conceptions trop cohérentes des ensembles sociotechniques. Ceci nous invite à abandonner l'histoire des énergies par types de source au profit d'une histoire focalisée sur les convertisseurs énergétiques en tant qu'artefacts technopolitiques.

\section{Articulation des métaphores, des types de détermination et des formes de temporalité}

La deuxième conclusion partielle est une synthèse historiographique articulant métaphores, déterminations et temporalités en histoire des techniques. Le choix des quatre métaphores analysées jusqu’à présent est motivé par leur usage courant en histoire des techniques, le flou conceptuel qui les entoure et un degré de complexité croissant des objets et phénomènes

56. Godelier, 1984, p. 9. 
en jeu. En effet, chaque nouvelle métaphore intègre un niveau supplémentaire de détermination et enrichit les temporalités concernées. Le tableau de synthèse ci-dessous détaille, pour chaque métaphore, cinq critères du domaine source : objets d'étude ; milieux de déploiement ; phénomènes (et disciplines) ; types de détermination (et donc niveaux d'explication) ; formes de temporalité.

Tableau de synthèse

\begin{tabular}{|c|c|c|c|c|c|}
\hline Métaphores & Objets & Milieux & Phénomènes & $\begin{array}{l}\text { Types } \\
\text { de détermina- } \\
\text { tion }\end{array}$ & $\begin{array}{l}\text { Formes } \\
\text { de tempo- } \\
\text { ralité }\end{array}$ \\
\hline trajectoire & $\begin{array}{l}\text { mobiles, } \\
\text { projectiles }\end{array}$ & espace vide & mécanique & $\begin{array}{l}\text { cause à effet } \\
\text { (déterminisme } \\
\text { strict) }\end{array}$ & $\begin{array}{l}\text { temps } \\
\text { opératoire }\end{array}$ \\
\hline transition & $\begin{array}{l}\text { population } \\
\text { de mobiles } \\
\text { en interac- } \\
\text { tion }\end{array}$ & $\begin{array}{l}\text { espace homo- } \\
\text { gène et fermé }\end{array}$ & $\begin{array}{l}\text { thermo- } \\
\text { dynamique }\end{array}$ & $\begin{array}{l}\text { corrélations } \\
\text { statistiques }\end{array}$ & $\begin{array}{l}\text { temps } \\
\text { de l'équilibre }\end{array}$ \\
\hline lignée & $\begin{array}{l}\text { organismes } \\
\text { vivants, } \\
\text { espèces } \\
\text { animales }\end{array}$ & $\begin{array}{l}\text { environ- } \\
\text { nement } \\
\text { hétérogène } \\
\text { et ouvert }\end{array}$ & biologique & $\begin{array}{l}\text { essais } \\
\text { et erreurs, } \\
\text { reproduction } \\
\text { et surgissement }\end{array}$ & $\begin{array}{l}\text { temps vécu, } \\
\text { mémoire de } \\
\text { l'expérience }\end{array}$ \\
\hline système & $\begin{array}{l}\text { ensembles } \\
\text { hommes- } \\
\text { machines } \\
\text { hétérogènes }\end{array}$ & $\begin{array}{l}\text { territoire } \\
\text { ouvert, } \\
\text { en interaction } \\
\text { avec d'autres }\end{array}$ & cybernétique & $\begin{array}{l}\text { auto-repro- } \\
\text { duction et } \\
\text { organisation, } \\
\text { émergence }\end{array}$ & $\begin{array}{l}\text { temps socio- } \\
\text { culturel, } \\
\text { champs } \\
\text { d'expérience }\end{array}$ \\
\hline
\end{tabular}

Chaque type de détermination peut être associé à des cas concrets en histoire des énergies. Pour une détermination mécanique : une machine à vapeur tire la chaîne d'une pompe à eau installée dans une mine. Pour une détermination statistique : «l'Étasunien moyen » consomme deux fois plus d'énergie que «l'Européen moyen ». Pour une détermination biologique : des animaux inventent, par des expériences répétées, des gestes économes en énergie au cours de leur vie, qui pourront être transmis (par imitation) aux descendants et aux voisins. Pour une détermination cybernétique : au $\mathrm{XVIII}^{\mathrm{e}}$ siècle, plusieurs acteurs techniques fabriquent, améliorent et vendent des «machines à feu ". Ces quatre exemples peuvent être distingués a posteriori dans le discours historien mais ne le sont pas a priori dans le processus historique. Il s'agit donc de penser les quatre types de détermination 
comme imbriqués dans n’importe quel « fait historique ${ }^{57}$ ». Ils sont rangés, en première lecture, du déterminisme à l'émergence, du plus simple au plus complexe, chaque type incorporant les précédents. Dans le même temps, les formes de temporalité se complexifient car elles se densifient en incorporant de plus en plus de relations conceptuelles et affectives au monde. Les deux dernières formes de temporalité en incorporant l'expérience vécue se distinguent ainsi des deux premières, qui n'ont accès qu'à l'expérimentation. Les deux derniers types de détermination incorporent une part d'intentionnalité, de projection des acteurs, qu'ils soient animaux ou humains, dans le champ d'expérience spatio-temporel du monde.

Cette hypothèse " animiste ${ }^{58}$ " vient rappeler une différence irréductible entre sciences de la nature et de l'homme : leurs rapports différents aux objets étudiés, aux niveaux cognitifs et affectifs. Les sciences de la nature forment une entreprise d'explication particulière du monde, basée sur des opérations mathématiques et expérimentales. Les sciences de l'ingénieur s’appuient sur ces opérativités pour mettre en place un rapport de contrôle matériel du monde naturel et vivant. L'explication matérielle et opératoire fait également partie des objectifs des sciences de l'homme et de la société. Ceci explique pourquoi les métaphores précédentes ne sont pas dénuées d'intérêt pour leur apport heuristique. Mais, les sciences de l'homme et de la société doivent, en outre, interpréter la signification du social et du culturel. Pour reprendre une distinction célèbre de Wilhelm Dilthey : "Nous expliquons la nature, nous comprenons la vie psychique ». Cette irréductibilité du social à des phénomènes matériels et biologiques rend toute analogie scientifique en histoire forcément limitée.

57. Nous suivons l'idée d'un holisme des causes historiques de Paul Veyne sans reprendre sa classification tripartite : «Résumons : l'explication historique pousse plus ou moins loin l'explication des facteurs ; par ailleurs, en ce monde sublunaire, ces facteurs sont de trois espèces. L'un est le hasard, qu'on appelle aussi causes superficielles, incident, génie ou occasion. L’autre s'appelle causes, ou conditions, ou données objectives : nous l'appellerons causes matérielles. Le dernier est la liberté, la délibération, que nous appellerons causes finales. Le moindre "fait" historique comporte ces trois éléments, s'il est humain. " (Veyne, 1996, p. 121).

58. "Le sens fort de la coupure [sujet-objet] est beaucoup plus central dans la pensée scientifique moderne, il postule que l'homme est le seul être doué d'intention dans la nature, que celle-ci est donc inerte, ou du moins simplement régie par des lois immuables, qu'elle n'est qu'une chose, matière amorphe. [... Au contraire,] l'hypothèse que, pour simplifier, on intitulera animiste semble bien plus plausible, à savoir : ce phénomène social est une intention en acte, il exprime en lui-même un projet, et une fois réalisé il s'épuise ou se dissout. " (Gras, 1979, p. 192). 
Ces considérations ont une conséquence d'ampleur pour l'épistémologie des sciences de l'homme et de la nature. Si la théorie sémantique des métaphores est si intéressante ici, c'est certes parce qu'elle a permis d'analyser les systèmes de correspondance sémantique mais aussi, et surtout, parce qu'elle a attiré notre attention sur la référence : le langage est à la fois description, inscription et représentation du monde. Si l'interprétation des temporalités historiques passe, de plus en plus souvent, par le filtre des métaphores des sciences de la nature, ceci implique également une représentation de plus en plus opératoire du temps. Or, ce modèle d'appréhension du temps ne constitue qu'une forme de temporalité historique. Il est donc important, pour ne pas réduire l'épistémologie de l'histoire à une perception trop mécanique, trop statistique ou trop évolutive, à renouveler les métaphores temporelles à partir d'autres domaines sources que les sciences de la nature. Réanimer les "métaphores mortes ", animer les "métaphores usées " et forger des " métaphores vives " sont autant de façons d' "inventer [...ce] qui implique à la fois découvrir et créer " des relations au monde par la médiation herméneutique ${ }^{59}$. Notre idée, pour finir, est de proposer deux nouvelles métaphores qui inscrivent l'histoire des techniques dans des pra330 tiques culturelles et sensibles.

\section{L'histoire des énergies comme «sac de nouds"}

Nous proposons, pour commencer, la métaphore du «sac de nœuds " pour étudier l'histoire des énergies au $\mathrm{xx}^{\mathrm{e}}$ siècle ${ }^{60}$. Une telle expression, d'ordre méthodologique et métaphorique, s'applique aux convertisseurs énergétiques. La métaphore du " nœud ${ }^{61}$ emprunte au domaine source du jeu - faire et défaire des nœuds - et le transfère au domaine cible du politique - comme carrefour géopolitique.

59. Ricœur, 1975 , p. 387-388.

60. Marrec et Teissier, 2018.

61. L'image d'une multitude de "nœuds " aux temporalités variées a déjà été utilisée par Michel Serres pour rendre compte de l'histoire des sciences comme " réseau multiple et complexe de chemins qui se chevauchent et s'entrecroisent en des nœuds, sommets ou carrefours, échangeurs où bifurquent deux ou plusieurs voies ", et où se joue une " multiplicité de temps différents " (Serres, 1989, p. 5). 
La métaphore du nœud établit quatre correspondances sémantiques entre le domaine source et les convertisseurs en histoire des énergies. Premièrement, les différents brins du nœud présentent une situation d'entrelacement de phénomènes, à la manière du "tissu sans coutures ", chaque phénomène pouvant incorporer plusieurs types de détermination, de temporalités et d'acteurs. La deuxième correspondance réside dans la difficulté à faire et à défaire un nœud, chaque nœud étant plus ou moins difficile à manœuvrer. Ces deux premières correspondances produisent une efficacité métaphorique au moins équivalente à celle du système sociotechnique en exprimant l'imbrication et l'inertie des ensembles ${ }^{62}$. Les deux derniers niveaux introduisent des correspondances supplémentaires, c'est-à-dire une intelligibilité nouvelle. La troisième correspondance souligne l'importance des cultures professionnelles dans la manière dont les brins d'un nœud sont choisis et agencés. Cette image permet de glisser du concept d'innovation à celui de "modes d'assemblage " comme "recomposition " permanente des techniques ${ }^{63}$. Ceci étend d'autant la compréhension des dynamiques techniques en rendant également légitimes les actions des usagers, des producteurs et des inventeurs. La quatrième correspondance se situe, non plus sur le nœud, mais dans le "sac ». La métaphore permet alors de sortir du registre $\mathrm{du}$ " système technique » du type " réseau électrique » car elle ne suppose pas a priori quels convertisseurs (nœuds) interagissent ensemble. Elle rend possible la rencontre de facteurs déterminants variés, qui ne sont ni hiérarchisés, ni univoques, ni toujours identiques.

Appliquons la métaphore au cas français des énergies éoliennes et solaires. Sur la période 1940-1960, le projet d'un aérogénérateur métropolitain est soutenu par la construction du réseau électrique au moment de la «modernisation " de la France à travers l'électrification du territoire national. En Algérie, au contraire, le projet de pompes éoliennes à destination des bergers nomades des hauts plateaux oranais s'appuie sur une politique coloniale pour la mise en valeur agricole de l'empire ${ }^{64}$. De même, sur la période 1950-1970, le programme des fours solaires d'Odeillo est

62. La matérialité complexe du nœud (deux brins ou plus, de nature différente, entrelacés de manière différente, selon une gestuelle plus ou moins rapide) traduit plus finement les possibilités diverses d'inertie et de fluidité sociotechniques que les deux paramètres du momentum (taille et vitesse).

63. Grimaud, Tastevin, Vidal, 2017.

64. Marrec, 2018, p. 378-388 et p. 435-456. 
principalement motivé par le contexte de compétition Est-Ouest de la guerre froide ${ }^{65}$ tandis que celui des pompes solaires de Dakar s'inscrit dans la politique internationale du " développement ${ }^{66}$ ". Davantage que des systèmes, ces quelques convertisseurs éoliens et solaires forment un « sac de nouds».

\section{L'histoire comme « psychologie des nénuphars »}

Du domaine du jeu, passons à celui de l'art en regardant un moment célèbre de l'histoire de la peinture : Les Nymphéas. De la fin des années 1880 au milieu des années 1920, Claude Monet peint environ 250 tableaux représentant des nénuphars dans un bassin, qui sont, pour l'essentiel dans son jardin à Giverny. Une telle entreprise, qui court sur trois décennies, rend compte d'une double série temporelle et discontinue : le changement, par étapes, au fil des heures, des jours et des saisons d'un paysage végétal (bassins, nénuphars et lumières) ; le changement existentiel et esthétique de la psychologie et de la sensualité du peintre. Les deux séries sont liées entre elles mais aucune n'est réductible à l'autre car chacune peut donner lieu à un surgissement imprévu de créativité : due à la coopération et à la compétition des nénuphars dans leur environnement pour la première ; due à l'existence et à la psychologie du peintre pour la seconde. La relation entre un élément de chaque série rend compte de ce que Félix Guattari a appelé " la vérité du lien » à partir de la psychanalyse ${ }^{67}$. Selon Guattari, ce lien est le support de dimensions humaines fondamentales telles que la création, l'éthique et l'esthétique.

Au fond, l'interrelation de ces deux séries hétérogènes de surgissement-créativité constitue une analogie pertinente pour expliquer ce que nous entendons par histoire. La première série constitue le processus historique si l'on reconnaît aux nénuphars une capacité de communication collective

65. Teissier, 2013.

66. Cooper, 2013.

67. S'opposant au "paradigme scientiste ", Félix Guattari propose de considérer, au lieu d'une vérité universelle, cette " vérité du rapport, une vérité de la relation, une unicité, une singularité qui va naître et qui va créer ce climat [...] de singularité existentielle, ce cachet de vérité qui fera que l'on n'est plus du tout dans un monde pseudo-scientifique, un monde technocratique, un monde de blouses blanches, un monde de causalité linéaire » (Guattari, 2005, p. 13-14). 
(que les sciences biologiques leur octroient depuis quelques années) et une psychologie individuelle (que les animistes leur octroient depuis quelques millénaires). La seconde série constitue le récit historique, tissé par les changements de savoir-faire, de sensibilité et d'état d'âme du peintre, les modes culturelles de son époque et les héritages de sa société. Nous appelons " psychologie des nénuphars " cette métaphore qui présente l'histoire dans sa dimension double, de processus et de récit. En insistant sur la double " psychologie », du peintre et des nénuphars, nous voulons introduire un cinquième type de détermination, propre à l'être humain, inscrit dans son expérience sensible du monde. Virginia Woolf appelle "l'intimité de l'âme ", dans Mrs Dalloway, cet espace individuel que "l'amour comme la religion ", s'efforcent de détruire par " conversion ». Elle file une métaphore aquatique pour opposer les profondeurs intimes aux surfaces sociales :

tout simplement bavarder. Car telle est la vérité sur notre âme, [...] notre moi intérieur, qui vit en eaux profondes comme un poisson et navigue dans les ténèbres, se frayant un chemin entre les fûts des algues géantes, passe sur des endroits où danse le soleil et s'enfonce encore dans les profondeurs lugubres, froides et impénétrables; parfois, elle jaillit à la surface et folâtre sur les vagues que ride le vent; car elle a un véritable besoin de se frotter, de se gratter, de s'enflammer grâce à ce bavardage. (Mrs Dalloway, p. 182-183)

Woolf considère les espaces intimes et sociaux comme nécessaires aux êtres humains selon les temps de la journée ou de la vie. Ce faisant, elle livre une clé de compréhension des cinq types de détermination que nous avons identifiés jusqu'ici : c'est par le va-et-vient du social à l'intime que l'individu se construit de manière singulière. C'est donc dans une interprétation relationnelle des cinq types de détermination et des cinq formes de temporalité qu'il faut penser l'histoire. En effet, c'est par opposition au temps de "la mesure » préconisé par la société (par le psychiatre William notamment) que les personnages du roman vivent leurs rythmes propres. Le temps vécu n'est pas un mais multiple, pas fixe mais interprété, pas universel mais ancré :

Big Ben sonna la demi-heure. [...] Au milieu des choses ordinaires, le battant tombait, tombait, rendant l'instant solennel [...] mais voici que l'autre horloge, l'horloge qui sonne toujours 
deux minutes après Big Ben, sonna en traînant $[\ldots$ v]olubile, agitée, [...] déversant plein de petits riens. Battus, brisés par les assauts des charrettes, la brutalité des camions, l'avide progression de myriades d'hommes anguleux, de femmes se pavanant, les dômes et les flèches des bureaux et des hôpitaux, les derniers petits riens semblaient se briser, comme les embruns d'une vague épuisée. (Mrs Dalloway, p. 147-149)

\section{Conclusion}

Nous avons attiré l'attention sur les limites et présupposés de quatre métaphores d'inspiration technoscientifique. De tels emplois sont courants et utiles, mais trop peu explicités en histoire des techniques. Ceci suggère que l'imaginaire des historiens est peuplé de concepts, d'images et de démons des sciences expérimentales et ingénieuriales. Un tel transfert est normal du fait de la fréquentation continue des acteurs des sciences et des techniques. Mais, il doit s'accompagner d'une réflexivité car les métaphores sont porteuses d'une réécriture permanente du monde. À trop puiser à la source mathématique et expérimentale, les imaginaires historiens pourraient s'habituer à penser les temporalités de manière trop opératoire, trop univoque, en oubliant les expériences et sensibilités. Nous avons montré comment au moins cinq formes de temporalité s'articulent à cinq types de détermination dans le processus et le récit historiques. Nous voulions contribuer à décoloniser nos esprits vis-à-vis de la rationalité moderne d'un temps universel et à mettre à distance l'idéologie envoûtante de la puissance technoscientifique des sociétés industrielles. Forger de nouvelles métaphores à partir d'activités ludiques ou culturelles - défaire un nœud ou plonger dans l'intimité de l'âme - est une voie stimulante pour s'affranchir du référentiel technoscientifique, tout en utilisant son heuristique. Ainsi, l'analyse critique et la création sémantique de métaphores fabriquent de nouvelles intelligibilités des présents que nous habitons, des passés que nous explorons et des futurs que nous envisageons. 


\section{Bibliographie}

Basalla Georges, "Énergie et civilisation », Culture technique, n 7, 1982, numéro spécial : Robotique, automation, p. 169-179.

Beltran Alain, "Conclusion ", dans Lamard Pierre et Stockopf Nicolas (dir.), La transition énergétique, un concept historique?, Presses du Septentrion, Villeneuve d'Ascq, 2018, p. 305-309.

Benjamin Walter, Sur le concept d'histoire, Édition de l'Éclat, Paris, 2014 [1940]. Blay Michel, Les Principia de Newton, PUF, Paris, 1995.

Boistel Guy et Le Gars Stéphane (dir.), Dans le champ solaire : Cartographie d'un objet scientifique, Hermann, Paris, 2015.

Bouvier Yves et Laborie Léonard, L'Europe en transitions : Énergie, mobilité, communication, XVIII-XXI siècles, Nouveau Monde, Paris, 2016.

Bouvier Yves et Pehlivanian Sophie (dir.), Annales historiques de l'électricité, $n^{\circ} 11,2013$, numéro spécial : Les politiques publiques de l'énergie solaire.

Bouvier Yves, "Les transitions énergétiques dans l'histoire, entre succession des techniques et sédimentation des enjeux ", dans Bouvier Yves (dir.), Les Défis énergétiques du XXI siècle, transition, concurrence et efficacité au prisme des sciences humaines, PIE Peter Lang, 2012, p. 23-36.

Chauvin-Michel Marion, Architecture solaire et politiques énergétiques en France de 1973 à 1985, thèse de doctorat, université Paris 1, 2012.

Cooper Frederic, "Conflits, réformes et décolonisation. La situation coloniale mise en cause ", dans SingaravéLOu Pierre (dir), Les Empires coloniaux, XIX XXe siècles, Points, Paris, 2013, p. 377-419.

Courbage Youssef et Todd Emmanuel, Le Rendez-vous des civilisations, Seuil, Paris, 2007.

DAVID Paul, "Understanding the Economies of QWERTY: The Necessity of History ", in Parker William N. (éd.), Economic History and the Modern Economist, Basil Blackwell, New York, 1986, p. 30-49.

Debeir Jean-Claude, Deléage Jean-Pierre, Hémery Daniel, Une Histoire de l'énergie. Les servitudes de la puissance, Flammarion, Paris, 2013.

Diop Thierno S., L’histoire de l'énergie solaire du Sénégal contemporain, mémoire de master 2 en histoire des techniques, université de Nantes, 2016.

Durkheim Émile, Formes élémentaires de la vie religieuse : le système totémique en Australie, PUF, Paris, 1912.

Ellul Jacques, Le Système technicien, Le cherche midi, Paris, 2012 [1977].

Foucault Michel, L'Archéologie du savoir, Gallimard, Paris, 1969.

Fressoz Jean-Baptiste, "Pour une histoire désorientée de l'énergie ", Entropia. L'histoire désorientée, $\mathrm{n}^{\circ}$ 15, 2013, p. 173-187.

Gille Bertrand (dir.), Histoire des techniques, La Pléiade, Paris, 1978. 
Godelier Maurice, L'Idéel et le matériel. Pensée, économies, sociétés, Flammarion, Paris, 1984.

Gras Alain, Fragilité de la puissance. Se libérer de l'emprise technologique, Fayard, Paris, 2003.

Gras Alain, Les Macro-systèmes techniques, PUF/Fayard, Paris, 1997.

Gras Alain, Sociologie des ruptures. Les pièges du temps en sciences sociales, PUF, Paris, 1979.

Grimaud Emmanuel, Tastevin Yann Philippe, Vidal Denis, "Low tech, hightech, wild tech. Réinventer la technologie ?" Techniques et Culture, nº 67, 2017, Low Tech? Wild Tech!, p. 12-29.

Guattari Félix, La philosophie est essentielle à l'existence humaine, Édition de l'Aube, La Tour d'Aigues, 2005.

Hеснт Gabrielle, Le Rayonnement de la France, énergie nucléaire et identité nationale après la Seconde Guerre mondiale, Édition Amsterdam, 2014.

HerléA Alexandre (dir.), L’Énergie solaire en France, Édition du CTHS, 1995.

Herring Emily, "Des évolutionnismes sans mécanisme, Les néolamarckismes métaphysiques d'Albert Vandel (1894-1980) et Pierre-Paul Grassé (18951985) ", Revue d'histoire des sciences, vol. 69, n 2, 2016, p. 369-398.

Hills Richard L., Power From Wind. A History of Windmill Technology, Cambridge University Press, Cambridge, 1996 [1994].

Hughes Thomas P., "L'histoire comme systèmes en évolution ", Annales. Histoire, Sciences Sociales, vol. 53, n 4-5, 1998, p. 839-857.

Hughes Thomas P., Networks of Power. Electrification in Western Society, 1880 1930, The Johns Hopkins University Press, Baltimore, 1986 [1983].

Hughes Thomas P., "Convergent Themes in the History of Science, Medicine, and Technology", Technology and Culture, vol. 22, n 3, 1981, p. 550-558.

Kryza Franz T., The Power of Light. The Epic Story of Man's Quest to Harness the Sun, Mac-Graw Hill, 2003.

Jarrige François et VRIGnON Alexis, "Colloque - Face à la puissance. Une histoire des énergies alternatives et renouvelables à l'âge industriel ( $\mathrm{XIX}^{\mathrm{e}}-\mathrm{XX}^{\mathrm{e}}$ siècles) ", Appel à communication, février 2017.

KosELleck Reinhardt, L'Expérience de l'histoire, Gallimard, Paris, trad. Alexandre Escudier, 1997.

Kövecses Zoltán, Metaphor. A Practical Introduction, Oxford University Press, Oxford, 2010.

Lakoff George, "The Contemporary Theory of Metaphor", in OrTONY Andrew (éd.), Metaphor and Thought, Cambridge University Press, Cambridge, 1993 [1979], p. 202-251.

Lakoff George and Johnson Mark, Metaphors We Live By, University of Chicago Press, Chicago, 1980. 
LAmard Pierre et Stoskopf Nicolas, "Introduction ", dans LAMARD Pierre et Stoskopf Nicolas (dir), La transition énergétique, un concept historique?, Presses du Septentrion, Villeneuve d'Ascq, 2018, p. 9-19.

Le Moigne Jean-Louis, "système », dans Lecourt Dominique (dir.), Dictionnaire d'histoire et de philosophie des sciences, PUF, Paris, 2003, p. 900-909.

Marrec Anaël, Histoire des énergies renouvelables en France, 1880-1990, thèse de doctorat, université de Nantes, 2018.

Marrec Anaël et Teissier Pierre, "Techno-politiques des énergies naturelles : énergies alternatives et politiques conservatrices des "trente glorieuses" ", Colloque Face à la puissance. Une histoire des énergies alternatives et renouvelables à l'àge industriel, université de Bourgogne et RUCHE, 22-23 mars 2018.

Pehlivanian Sophie, Histoire de l'énergie solaire en France : science, technologies et patrimoine d'une filière d'avenir, thèse de doctorat, université de Grenoble, 2014.

Quemada Bernard, "Technique et langage », dans Gille Bertrand (dir.), Histoire des techniques, bibliothèque de La Pléiade, 1978, p. 1146-1240.

Ricceur Paul, La Métaphore vive, Seuil, Paris, 1975.

Righter Robert W., Wind Energy in America. A History, University of Oklahoma Press, 2008 [1996].

Serres Michel, "Préface ", dans Serres Michel (dir.), Eléments d'histoire des sciences, Bordas, Paris, 1989, p. 1-15.

TeIssier Pierre, "Le solaire passif à l'ombre de la politique énergétique française, 1945-1986 ", dans Bouvier Yves et Pehlivanian Sophie (dir.), Annales historiques de l'électricité, $\mathrm{n}^{\circ}$ 11, 2013, Les politiques publiques de l'énergie solaire, p. 11-25.

Veyne Paul, Comment on écrit l'histoire? Essai d'épistémologie, Seuil, Paris, 1996 [1971].

Woolf, Virginia, Mrs Dalloway, Le Livre de Poche, Paris, trad. Pascale Michon, 1993 [1925]. 


\section{Les auteurs}

Pierre Teissier est maître de conférences en épistémologie, histoire des sciences et des techniques au Centre François Viète de l'université de Nantes. Il étudie les sciences et les techniques à l'époque contemporaine à travers deux domaines spécifiques : les machines et les énergies (énergie solaire, piles à combustible, voitures électriques) ; la chimie et les matériaux. Contact : pierre.teissier@univnantes.fr

Anaël Marrec est docteure en histoire des sciences et des techniques, chercheuse associée au Centre François Viète de l'université de Nantes. Elle a soutenu une thèse sur l'histoire des énergies renouvelables en France de 1880 à 1990 et travaille actuellement sur l'histoire des énergies à l'époque contemporaine. Publications : Histoire des énergies renouvelables en France (1880-1990), thèse de doctorat, université de Nantes, 2018 ; « Les îles industrielles dans l'histoire des énergies renouvelables. Récurrence d'un projet », dans LAMARD Pierre et STOSKOPF Nicolas, La transition énergétique, un concept historique ?, Presses du Septentrion, Villeneuve d’Ascq, 2018, p. 177-195; «Politiques des forces naturelles et imaginaires de l'énergie pendant l'entre-deux-guerres », Pour Mémoire, n 18, 2016

p.83-92.Contact : anael.marrec@univ-nantes.fr 